\title{
Clinical utility of robot-assisted radical prostatectomy: advances and current status
}

\author{
This article was published in the following Dove Press journal: \\ Robotic Surgery: Research and Reviews \\ 12 March 2015 \\ Number of times this article has been viewed
}

\section{Paul Sturch \\ Nicholas T Raison \\ Ben Challacombe}

Urology department, Guy's Hospital, Guy's and St Thomas' Hospital Trust, London, UK

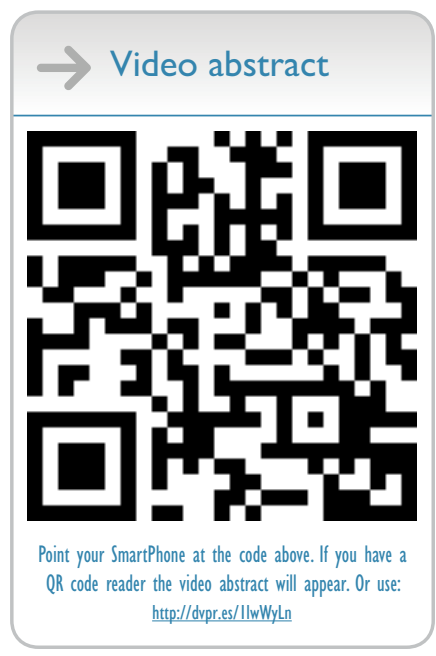

Correspondence: Ben Challacombe Guy's Hospital, Great Maze Pond, London, UK, SEI 9RT

Email benchallacombe@doctors.org.uk
Abstract: The development of robotic technology in surgery has changed the landscape of radical surgery for localized prostate cancer. Early on, urologists recognizing its advantages were quick to adopt robotic techniques, building on the experience gained from years of open prostatectomy. The 3D HD vision and the precision endowrist instruments suit delicate nerve sparing surgery and facilitate excellent functional as well as oncological outcomes. Robotassisted radical prostatectomy evolved from open and laparoscopic techniques and then advanced alongside developments in robotic hardware. We describe the evolution of robotic prostate surgery, advantages and disadvantages of the techniques and explore the potential future of robotic technology in prostate surgery.

Keywords: prostatectomy, prostate cancer, robot-assisted radical prostatectomy, RARP

\section{Introduction}

Prostate cancer $(\mathrm{CaP})$ remains one of the most commonly diagnosed solid organ malignancies in developed countries. From 2000 to 2003, the age-adjusted incidence rate was $28.5 / 100,000$ men in the $\mathrm{USA}^{1}$ and 27.2/100,000 in 2001 in the UK. ${ }^{2}$

The first radical retropubic prostatectomy (RRP) was performed by Terence Millin in 1946. ${ }^{3}$ Subsequent advances in the understanding of pelvic anatomy and surgical technique by Walsh ${ }^{4}$ led to open radical prostatectomy (RP) becoming the gold standard and most widely used operation in the surgical management of localized $\mathrm{CaP}$.

Meanwhile, the development of robotic technologies in surgery was picking up speed. From the first applications of robots in urology with the PROBOT for transurethral resection of the prostate in the $1980 \mathrm{~s},{ }^{5}$ urologists have been at the forefront of developing new robotic technology.

The Californian company Computer Motion (Goleta, CA, USA) developed the first robotic-assisted laparoscopic system: the Automated Endoscopic System for Optimal Positioning (AESOP). ${ }^{6}$ This was used successfully for laparoscopic RP (LRP) with the voice- or pedal-controlled camera providing steadier images than human assistants. ${ }^{7}$

Master-slave operating robots, developed in the 1990s, represented the next major advance. ${ }^{8}$ The ZEUS system developed by Computer Motion incorporated the AESOP voice-controlled laparoscopic camera with a master-slave system, where movements of two joystick controllers were remotely transmitted to laparoscopic instruments attached to the operating table.

The ZEUS system was initially a rival to, and has now been superseded by, the da Vinci Surgical System (Intuitive Surgical, Mountain View, CA, USA), which uses a portable base to mount the three-dimensional imaging system and instruments 
separate to the operating table. The da Vinci System is operated by a surgeon sitting at a remote console and whose movements are replicated with six degrees of freedom in the 'endowrist' instruments. The $3 \mathrm{D}$ camera system also provides 10-15 times magnification, tremor elimination, and motion scaling. ${ }^{9}$

The da Vinci System was first introduced in 1999 and gained US Food and Drug Administration approval for clinical use in 2000. Following a merger with Computer Motion in 2003, Intuitive became the sole producer of robotic surgical devices. ${ }^{10}$

In 2006, Intuitive introduced its third-generation model, the da Vinci S System, with a fourth system, the Xi launched in 2014. New generation systems incorporated a less bulky bedside platform, with improved range of motion and easier docking, along with an additional fourth arm upgrade. ${ }^{11}$

The da Vinci System has found popularity within the urological community who operate for long periods deep within the pelvis where steady, magnified, 3D, HD views and precision instruments come into their own and are all certainly reasons as to why robot-assisted surgery has become so widespread in urology institutes worldwide. There are now over 2,000 systems in use worldwide, with robotics developed in every continent other than the Antarctic.

In the USA, the proportion of radical prostatectomies carried out with the use of robot-assisted surgery continues to increase. In 2006, a total of $41 \%$ of RPs were robot-assisted radical prostatectomy (RARP); in 2007, this number rose to more than $60 \%$ and to more than $80 \%$ in $2008 .^{12}$

\section{Evolution of the technique}

The Vattikuti Institute in Detroit, with Dr Mani Menon as lead robotic pioneer, has been instrumental in the evolution of techniques for RARP since 2000. The Vattikuti Institute Prostatectomy (VIP) applies the technological advantages of the da Vinci system to the anatomical technique for open prostatectomy described by $\mathrm{Walsh}^{4}$ and the laparoscopic prostatectomy technique described by Guillonneau and Vallancien ${ }^{13}$ at the Montsouris Institute in Paris.

One major difference between laparoscopic prostatectomy and RARP is the separation of the two operating surgeons: one at the console and the other at the patient's side. Both surgeons of the Detroit team spent a month training in the laparoscopic technique with the Montsouris team, working on animal models and observing laparoscopic cases. The French team then trained the Detroit team in a further 30 laparoscopic cases at the Vattikuti institute before training on the da Vinci robot began. This involved in vitro exercises in dissection, suturing, and manipulation of the robotic instruments, as well as wet-lab sessions with pig models, and fresh human cadavers.

\section{VIP technique described for first 30 patients \\ Patient positioning}

The patient is initially placed in the supine position on the operating table. ${ }^{14}$ Padding is placed over pressure points on shoulders, back, arms, and legs, with additional gel pads placed under the patient's back. The hands are carefully wrapped and tucked alongside the patient. Care is taken to pad any area that comes in contact with the operating table or that may come in contact with the robot, to reduce the risk of neuropraxia. Two straps are crossed from the shoulders across the patient's chest to reduce the chance of slipping when the table is tilted. Rigid shoulder supports are avoided due to the risk of nerve injury. Once secure, the legs are placed in the lithotomy position (or frog leg for patients under 6 feet in height). The abdomen is shaved, prepped, and draped, and the table is angulated to steep Trendelenburg.

\section{Pneumoperitoneum and port position}

The VIP team establish a pneumoperitoneum using a Veress needle inserted via a left upper abdominal or umbilical puncture. Carbon dioxide is insufflated to a pressure of 12-15 mmHg.

A $12 \mathrm{~mm}$ umbilical port is introduced and subsequent ports are sited under vision. Two $8 \mathrm{~mm}$ robot ports are placed $10 \mathrm{~cm}$ lateral to the midline on a line between the umbilicus and anterior superior iliac spine. Two further assistant ports for suction and a laparoscopic instrument are placed on the right side of the abdomen. A sixth $5 \mathrm{~mm}$ assistant port is placed laterally, inferior to the left-sided robotic port. The robot is then carefully docked in a position between the patient's raised legs.

The patient-side surgeon uses normal laparoscopic instruments to assist the console surgeon's monopolar hook and forceps. These are switched to two needle drivers for the anastomosis.

\section{Surgical steps}

If indicated, a lymph node dissection is carried out using a $30^{\circ}$ down lens using 1:3 scaling to allow a wide field of vision and precise dissection.

The Montsouris technique begins with retrovesical dissection of the seminal vesicle and vas deferens. In contrast, the VIP technique starts with anterior dissection of the parietal 
peritoneum overlying to bladder to allow access to the vasa, which are then followed inferoposteriorly to the seminal vesicles. A $30^{\circ}$ down lens is used for this. The seminal vesicle pedicles and artery of the vas are controlled with bipolar diathermy or a clip. In two further adaptations of the Montsouris technique, the vas is not transected until the posterior surface of the seminal vesicle has been identified, to avoid inadvertent damage to the ureter, and the fascia overlying the seminal vesicle is opened anteriorly to allow easier delivery of the vesicles and vas later in the operation.

The camera is then changed back to a $0^{\circ}$ lens with a $1: 1$ non-scaling view for mobilization of the bladder. Scaling is changed to 1:3 to sweep tissue away from the pubic symphysis, expose the endopelvic fascia, and clear the apex of the prostate. Bipolar diathermy is used for hemostasis of tributary vessels in this region. A $0^{\circ}$ or $30^{\circ}$ up lens is used for the dorsal vein stitch, which is carried out using a similar technique to that of the laparoscopic procedure.

For dissection of the bladder neck a monopolar hook is used with a $30^{\circ}$ down lens. Once the urethral catheter is exposed, it is deflated and delivered anteriorly to expose the posterior bladder neck, which is then divided with the hook. The vasa and seminal vesicles are then lifted up and the lateral pedicles exposed.

The lateral pedicles are dissected using a combination of blunt and sharp dissection and controlled using Hem-o-Lok clips (Weck Systems, Triangle Park, NC, USA). The dissection is carried down as far as possible following the curvature of the prostate, using a nerve-sparing technique if indicated.

Using the seminal vesicles and vasa to lift the prostate, Denonvillier's fascia is incised and dissected off the rectum down to the prostatic apex.

Switching back to a $0^{\circ}$ lens, the dorsal venous complex is divided with a diathermy hook and the anterior urethral wall exposed. To identify the midline of the urethral wall, a urethral sound is inserted to guide the transection with scissors. This is carried out just distal to the apex of the prostate, with the aim of reducing the chance of a positive margin. The posterior wall of the urethra is transected and the specimen bagged in an EndoCatch (Ethicon, Somerville, NJ, USA) retrieval bag.

The vesicourethral anastomosis is constructed using an interrupted or running stitch depending on whether the bladder neck has been preserved or is particularly wide. The Van Velthoven single knot running suture is most commonly used. ${ }^{15}$ It allows a simple, watertight anastomosis to be made with a single intracorporeal knot. The da Vinci Endowrist system has significant advantages over laparoscopic instruments in completing this important step in the procedure. Sutures can be placed at almost any angle, and continuous suturing is relatively straightforward. Various techniques, such as anterior, posterior, or complete reconstructions of the vesicourethral junction, have been described without any single technique being shown to be superior. ${ }^{16}$

A 20F Foley catheter is inserted across the anastomosis and inflated with $10-15 \mathrm{~mL}$, and a leak test with $250 \mathrm{~mL}$ saline is carried out. A suction drain is left in situ.

Post-operatively, the patient is started on clear fluids the evening of the surgery and can start eating once flatus has been passed. The drain is removed after 24 hours, and the catheter removed on post-operative day 4 following a cystogram.

\section{Evolution of the technique}

Since the initial description, there has been a progressive evolution of the RARP. Different approaches have been developed, such as extraperitoneal and retrograde techniques. The extraperitoneal RARP was born out of experience from laparoscopic extraperitoneal surgery. ${ }^{17}$ Whilst it is less common than the transperitoneal technique, it does confer important benefits. Staying outside the peritoneum reduces the risk of intra-abdominal complications such as bowel injury. Additionally, there is less need for a steep Trendelenburg position, which is advantageous in obese patients, but comes at the expense of a reduced working space.$^{18}$ Extended pelvic lymphadenectomy is more difficult and may not be possible to the same extent.

Retrograde RARP is not commonly performed but replicates the RRP described by Walsh, ${ }^{4}$ with retrograde dissection of the prostate and neurovascular bundles. By replicating this technique, it aims to facilitate the adoption of RARP by surgeons experienced in performing open prostatectomies. Retrograde dissection has also been shown to offer higher potency rates than an anterograde approach. ${ }^{19}$

\section{The dorsal venous complex}

In 2004, a team from the University of California developed a new technique for apical dissection and stapled control of the dorsal venous complex (DVC). They had previously used the VIP technique of two 'figure of eight' sutures at the apex to control the DVC followed by electrocautery to divide the DVC and urethra. They were concerned that bunching of tissue between the sutures distorted the anatomy leading to higher positive apical margin rates.

They describe a new technique involving the careful dissection of all fat overlying the puboprostatic ligaments and 
DVC until the entire surface of the DVC is exposed. The puboprostatic ligament and levator fibers attached to the DVC are also dissected free utilizing the precise dissection of the Endowrist. This exposes a greater length of DVC, allowing stapling across the complex with a $45 \mathrm{~mm}$ Endo-GIA stapler. A catheter protects the urethra from being stapled. This technique was shown to reduce apical positive margin rate for pT2 tumors from $27.3 \%$ to $4.7 \%{ }^{20}$ Currently, most centers are moving towards 'cold cutting' the DVC with scissors after the entire prostate has been mobilized with the pneumo peritoneum pressure briefly elevated to prevent venous bleeding. This may allow a more precise apical dissection, preserving urethral length whilst optimizing apical margin rates. These improvements are countered by higher blood loss. ${ }^{21}$

Subsequent refinements in RARP have focused on improving functional outcomes though development of nerve-sparing techniques.

By using the magnification of the da Vinci System, the VIP team were able to clearly delineate the layers of the prostatic fascia. This allowed preservation of the nerves and vessels running between the inner, prostatic, and outer levator fascia. The assistant facilitates the dissection by retracting the prostate in a superiomedial direction and retracting tissues adjacent to the neurovascular bundle laterally. This allows the console surgeon to enter a plane between the prostate capsule and overlying prostatic fascia. The space is developed with articulated scissors for blunt dissection and sparing use of bipolar electrocautery. The fascia is gently pushed off the surface of the prostate from the base down to the apex where the fascia fuses with the puboprostatic ligament, capsule and veins. Kaul et al ${ }^{22}$ have described this high fascial release with the resultant sheath of hanging fascia as the "Veil of Aphrodite." This technique showed promising results in a series of 35 patients when compared with 23 patients undergoing the standard nerve-sparing technique, all for localized CaP with normal erections (Sexual Health Inventory for Men [SHIM] score $>21$ unaided). Significantly more "veil" patients had erections strong enough for intercourse (97\% vs 74\%) and had a SHIM score $>21(86 \%$ vs $26 \%){ }^{23}$

In the following year, Ahlering et al described a nervepreserving procedure without the use of surgical clips or electrocautery. ${ }^{24}$ Instead, after delineation of the vascular pedicles, laparoscopic bulldog clips are applied and the pedicles are dissected close to the prostate. The neurovascular bundle is then gently dissected off the prostate, and FloSeal (Baxter, Irvine, CA, USA) is applied along the length of dissected tissue. A hemostatic layer of Gelfoam is applied on top of the FloSeal. The bulldog clips are removed after the prostate is dissected free anteriorly, and any bleeding from the pedicles is controlled with individual sutures. In their series of 17 patients, four required hemostatic sutures. ${ }^{24}$

Menon et al published further adaptations to their evolving experience in $2007^{25}$ and $2009 .{ }^{26}$ They describe a refinement of the veil technique where the interfascial dissection is continued anteriorly, preserving the prostatic fascia in between the 11 o'clock and 1 o'clock positions where it is adherent to the capsule. Careful dissection in this way can create an avascular hood separating the dorsal vein complex. They also used suprapubic catheterization, inserted under vision intraoperatively instead of a urethral catheter to decrease urethral discomfort following surgery. The final modification they describe is a limited lymph node dissection centered on internal iliac and obturator but excluding the external iliac nodes in low- and intermediaterisk disease. Their data suggest an improvement in erectile function, with $94 \%$ of patients able to achieve sexual intercourse between 6 and 18 months following surgery, a low incidence of postoperative catheter discomfort, and a higher yield of positive nodes, although fewer nodes were harvested overall. ${ }^{26}$

\section{Oncological and functional outcomes of RARP}

Whilst RARP is now widely recognized as the gold standard surgical treatment for localized $\mathrm{CaP}$, its superiority in regards to outcomes and complications remains contested. Objective assessment is made difficult by the lack of highlevel research. The majority of the literature is composed of single-center experiences, and the majority of the comparative studies are let down by poor methodologies. Combined with limited long-term follow-up data, accurate assessment of outcomes is difficult. Numerous studies have shown that RARP results in fewer perioperative complications, but, when adjusted for covariates, the majority do not reach significance. The only parameters in which RARP is persistently shown to offer an advantage is blood loss and transfusion rates, with very low rates of bladder neck stenosis compared with the laparoscopic interrupted anastomotic technique. ${ }^{16}$

A successful outcome following CaP surgery is judged not only by oncological cure, but also the impact on the patient's quality of life. The two most important functional outcomes following RP are continence and erectile function.

The positive surgical margin (PSM) rate is commonly used as a surrogate marker for long-term oncological outcomes. Even then, only a minority of papers have published 
PSM rates; a meta-analysis by Ficarra et $\mathrm{al}^{27}$ was only able to include eleven studies in their analysis of oncological data. As a result, comparisons of oncological outcomes between RARP and LRP/RRP remain contentious. Ficarra et $\mathrm{al}^{28}$ showed significant advantages for RARP over RRP in regards to PSM whilst RARP versus LRP were equal. On the other hand, Alemozaffar et al $^{29}$ demonstrated no difference in either PSM or biochemical recurrence (BCR) at 3 and 5 years between RARP and RRP. One of the largest studies analyzed over 50,000 LRP and 60,000 RARP. ${ }^{30}$ Interestingly, whilst they found that overall and pT2 PSM rates were significantly lower in RARP than in LRP, these differences were lost with propensity adjustment.

A number of aspects of robotic surgery have been identified that influence PSM rates. Surgeon experience has been analyzed in a number of trials, and although an improvement was seen with increasing experience for pT2 cancers, this was not replicated in overall PSM rates, which remained stable. ${ }^{16}$ However, fellowship-trained robotic surgeons were shown to be superior to colleagues who had moved from open surgery.

Furthermore, a PSM does not necessarily lead to BCR, and it is not clear whether the possible superiority of PSM rates with RARP translates into improved long-term oncological results. Reports of short- ${ }^{31}$ and mid-term ${ }^{32,33} \mathrm{BCR}$ rates have been largely comparable between RRP, LRP, and RARP. Magheli et $\mathrm{al}^{33}$ found that, while RARP had a lower PSM than either RRP or LRP, there was no difference in the $B C R$ rate. Similarly, Novara et a $1^{16,34}$ showed equal results for BCR-free survival (BCRFS) rates between RRP and RARP or RARP and LRP.

The primary factors associated with improved oncological outcome appear to be the disease rather than surgical technique. Preoperative prostate-specific antigen (PSA), Gleason score, and postoperative margin status were the most indicative factors for BCRFS.

Recent changes to the diagnosis and management of $\mathrm{CaP}$ through PSA screening and greater public awareness have led to a lower stage disease at presentation that is potentially easier to treat. Results from these patients may skew outcome data. In contrast, high-volume tertiary referral centers have tended to take on patients with moderate- to high-risk disease as their techniques develop. This presents further challenges when directly comparing outcome data. The Vattikuti Institute has been able to publish long-term follow-up data for almost 5,000 patients, showing a BCRFS of $81 \%$ at 8 years, with a Gleason score $\geq 3+4$ in $68 \%$ of patients and $\geq$ pT3a disease in $34 \%{ }^{35}$
Urinary incontinence is one of the most devastating complications following RP, with a significant impact on quality of life. Excessive traction on the urethra and disturbance of the pelvic floor musculature were felt to be likely factors influencing early continence rates following RRP and LRP, and a number of surgical techniques such as posterior reconstruction of Denonvillier's musculofascial plate and reconstruction of the urethra were introduced ${ }^{36}$ Accurate assessment of continence rates has been severely hampered by a lack of precise definitions of continence, the sparse use of validated questionnaires, and the lack of randomized studies. Again there is considerable variability in outcome data; however, systematic reviews of recent single-center trials do show that RARP is at least as good as RRP, if not better. ${ }^{37}$

Among the various quality of life outcomes associated with RP, sexual function in previously potent men is most closely related to positive quality of life outcomes and the most commonly impaired. Athermal and extended nervesparing techniques have been made possible with RARP. As with the other components of the trifecta, a lack of prospective comparative trials and infrequent use of validated questionnaires (eg, International Index of Erectile Function) make analysis difficult. Mottrie et al only identified two papers that used validated questionnaires, although cumulative data did suggest a benefit with RARP. ${ }^{38}$

However, Porpiglia et al ${ }^{39}$ did report more favorable functional outcomes in patients undergoing RARP than in those undergoing LRP, with no difference in oncological outcomes between the groups. In their prospective randomized control study of 120 patients operated on by a single surgeon, RARP was shown to provide statistically greater continence rates from the point of catheter removal onwards than was LRP. Continence rates at 1 year were $95 \%$ following RARP compared with $83.3 \%$ with LRP $(P=0.04)$. Recovery of sexual function in patients who underwent a nerve sparing procedure was in greater in the RARP group at 12 months compared to LRP ( $80 \%$ vs $54.2 \%)$.

Despite the potential advantages of RARP, the cost premium for the technology has limited its uptake. High fixed capital and maintenance costs mean that robotic surgery is likely to remain expensive in comparison with laparoscopic or open alternatives. A number of potential measures have been shown to reduce excess expenses. Maintaining a high throughput of cases improves the cost effectiveness of each case; centers performing $>150$ cases per year were able to reduce the incremental cost effectiveness ratio by $£ 17,822$ compared with centers performing $<100$ cases per year. ${ }^{40}$ Length of stay, operating room time, and more economical use of disposable 
robotic equipment such as suction devices can also reduce excess costs. ${ }^{41}$ In this way, Rebuck et al $^{42}$ were able to cut the cost of an RARP by 27\% (\$US1638). Yet a major factor in assessing the cost effectiveness of RARP is the improvement in PSM and long-term outcomes. As argued above, this relationship is yet to be proven; therefore, these economic forecasts need to be assessed with a degree of caution.

\section{Where does the future of robotic prostatectomy lie?}

Following the explosive entry of robotic surgery into the field of urology almost 15 years ago, recent advances in technology have slowed. Dedicated robotic theatres are being constructed hard-wired for robotic systems with the facility to ceiling mount instruments. ${ }^{43}$ The next generation of the da Vinci System, the Xi, facilitates multi-quadrant use with improved optics and simulation but does not change the fundamentals of the earlier machines.

The inclusion of haptic technology providing tactile feedback may provide an additional advantage to the operating surgeon; however, with the high quality of virtual operative simulator training available on the da Vinci System, and the advantages of the three-dimensional, high-definition and magnified visuals, new robotic surgeons are less reliant on tactile feedback.

The integration of augmented reality cross-sectional imaging with the da Vinci imaging system is a future development that will guide intraoperative dissection, and, when combined with thermal or fluorescent real-time imaging techniques, may aid the quality of nerve-sparing oncological outcomes and targeted lymph node dissection. ${ }^{44}$

As with all new technology, the natural drive is a reduction in size with each following generation. Nanotechnology; materials, devices, and systems measured in nanometers is an exciting and developing field in urology. Nanoparticles that can be employed to deliver therapeutic agents to targeted tissues have a potential role in the treatment of $\mathrm{CaP} .{ }^{45} \mathrm{~A}$ further role in the management of $\mathrm{CaP}$ is the identification of involved lymph nodes which, in addition to a primary diagnostic benefit, when combined with fluorescence and pre-operative imaging overlay, may aid lymph node dissection at RARP.

Future developments in nanotechnology applied to urology are eagerly awaited, and tools such as nanotweezers for micro-surgical procedures may herald the development of new technology for nerve preservation in RARP. ${ }^{46}$

\section{Disclosure}

The authors report no conflicts of interest in this work.

\section{References}

1. Howlader N, Noone AM, Krapcho M, et al., eds. SEER Cancer Statistics Review, 1975-2011. Bethesda, MD; 2014. Available from: http://www. seer.cancer.gov/csr/1975_2011/. Accessed April 2014.

2. Quinn M, Babb P, Brock A, Kirby L, Jones J. Cancer Trends in England and Wales, 1950-1999: Studies on Medical and Population Subjects No 66. London: Stationery Office; 2001.

3. Millin T. Retropubic prostatectomy. Br J Urol. 1946;18:33-38.

4. Walsh PC. Anatomic radical prostatectomy: evolution of the surgical technique. J Urol. 1998;160(6 Pt 2):2418-2424.

5. Shah J, Mackay S, Rockall T, Vale J, Darzi A. 'Urobotics': robots in urology. BJU Int. 2001;88(4):313-320.

6. Nedas TG, Challacombe BJ, Dasgupta P. Robotics in urology: an update. Int J Med Robot. 2005;1(2):13-18.

7. Kavoussi LR, Moore RG, Adams JB, Partin AW. Comparison of robotic versus human laparoscopic camera control. J Urol. 1995;154(6): 2134-2136.

8. Bowersox JC, Cornum RL. Remote operative urology using a surgical telemanipulator system: preliminary observations. Urology. 1998;52(1):17-22.

9. Dharia SP, Falcone T. Robotics in reproductive medicine. Fertil Steril. 2005;84(1):1-11.

10. Yates DR, Vaessen C, Roupret M. From Leonardo to da Vinci: the history of robot-assisted surgery in urology. BJU Int. 2011;108(11): 1708-1713; discussion 1714.

11. Shah K, Abaza R. Comparison of intraoperative outcomes using the new and old generation da Vinci ${ }^{\circledR}$ robot for robot-assisted laparoscopic prostatectomy. BJU Int. 2011;108(10):1642-1645.

12. Mavrich Villavicencio H, Esquena S, Palou Redorta J, Gómez Ruíz JJ. [Robotic radical prostatectomy: overview of our learning curve]. Actas Urol Esp. 2007;31(6):587-592. Spanish [with English abstract].

13. Guillonneau B, Vallancien G. Laparoscopic radical prostatectomy: the Montsouris technique. J Urol. 2000;163(6):1643-1649.

14. Tewari A, Peabody J, Sarle R, et al. Technique of da Vinci robot-assisted anatomic radical prostatectomy. Urology. 2002;60(4):569-572.

15. Van Velthoven RF, Ahlering TE, Peltier A, Skarecky DW, Clayman RV. Technique for laparoscopic running urethrovesical anastomosis: the single knot method. Urology. 2003;61(4):699-702.

16. Novara G, Ficarra V, Rosen RC, et al. Systematic review and metaanalysis of perioperative outcomes and complications after robotassisted radical prostatectomy. Eur Urol. 2012;62(3):431-452.

17. Gettman MT, Hoznek A, Salomon L, et al. Laparoscopic radical prostatectomy: description of the extraperitoneal approach using the da Vinci robotic system. J Urol. 2003;170(2 Pt 1):416-419.

18. Atug F, Castle EP, Woods M, Srivastav SK, Thomas R, Davis R. Transperitoneal versus extraperitoneal robotic-assisted radical prostatectomy: is one better than the other? Urology. 2006;68(5): 1077-1081.

19. Vricella GJ, Klaassen Z, Terris MK, Madi R. Retrograde robotic radical prostatectomy: description of a new technique and early perioperative outcomes. ISRN Urol. 2014;2014:945604.

20. Ahlering TE, Eichel L, Edwards RA, Lee DI, Skarecky DW. Robotic radical prostatectomy: a technique to reduce pT2 positive margins. Urology. 2004;64(6):1224-1228.

21. Lei Y, Alemozaffar M, Williams SB, et al. Athermal division and selective suture ligation of the dorsal vein complex during robotassisted laparoscopic radical prostatectomy: description of technique and outcomes. Eur Urol. 2011;59(2):235-243.

22. Kaul S, Savera A, Badani K, Fumo M, Bhandari A, Menon M. Functional outcomes and oncological efficacy of Vattikuti Institute prostatectomy with Veil of Aphrodite nerve-sparing: an analysis of 154 consecutive patients. BJU Int. 2006;97(3):467-472.

23. Kaul S, Bhandari A, Hemal A, Savera A, Shrivastava A, Menon M. Robotic radical prostatectomy with preservation of the prostatic fascia: a feasibility study. Urology. 2005;66(6):1261-1265.

24. Ahlering TE, Eichel L, Chou D, Skarecky DW. Feasibility study for robotic radical prostatectomy cautery-free neurovascular bundle preservation. Urology. 2005;65(5):994-997. 
25. Menon M, Shrivastava A, Kaul S, et al. Vattikuti Institute prostatectomy: contemporary technique and analysis of results. Eur Urol. 2007;51(3): 648-657; discussion 657-658.

26. Menon M, Shrivastava A, Bhandari M, Satyanarayana R, Siva S, Agarwal PK. Vattikuti Institute prostatectomy: technical modifications in 2009. Eur Urol. 2009;56(1):89-96.

27. Ficarra V, Cavalleri S, Novara G, Aragona M, Artibani W. Evidence from robot-assisted laparoscopic radical prostatectomy: a systematic review. Eur Urol. 2007;51(1):45-55; discussion 56.

28. Ficarra V, Novara G, Artibani W, et al. Retropubic, laparoscopic, and robot-assisted radical prostatectomy: a systematic review and cumulative analysis of comparative studies. Eur Urol. 2009;55(5):1037-1063.

29. Alemozaffar M, Sanda M, Yecies D, Mucci LA, Stampfer MJ, Kenfield SA. Benchmarks for operative outcomes of robotic and open radical prostatectomy: results from the Health Professionals Follow-up study. Eur Urol. Epub February 11, 2014.

30. Tewari A, Sooriakumaran P, Bloch DA, Seshadri-Kreaden U, Hebert AE, Wiklund $\mathrm{P}$. Positive surgical margin and perioperative complication rates of primary surgical treatments for prostate cancer: a systematic review and meta-analysis comparing retropubic, laparoscopic, and robotic prostatectomy. Eur Urol. 2012;62(1):1-15.

31. Barocas DA, Salem S, Kordan Y, et al. Robotic assisted laparoscopic prostatectomy versus radical retropubic prostatectomy for clinically localized prostate cancer: comparison of short-term biochemical recurrence-free survival. J Urol. 2010;183(3):990-996.

32. Drouin SJ, Vaessen C, Hupertan V, et al. Comparison of mid-term carcinologic control obtained after open, laparoscopic, and robot-assisted radical prostatectomy for localized prostate cancer. World $J$ Urol. 2009;27(5):599-605.

33. Magheli A, Gonzalgo ML, Su LM, et al. Impact of surgical technique (open vs laparoscopic vs robotic-assisted) on pathological and biochemical outcomes following radical prostatectomy: an analysis using propensity score matching. BJU Int. 2011;107(12):1956-1962.

34. Novara G, Ficarra V, Mocellin S, et al. Systematic review and metaanalysis of studies reporting oncologic outcome after robot-assisted radical prostatectomy. Eur Urol. 2012;62(3):382-404
35. Sukumar S, Rogers CG, Trinh Q-D, et al. Oncological outcomes after robot-assisted radical prostatectomy: long term follow-up in 4,803 patients. BJU Int. Epub August 12, 2013.

36. Dasgupta P, Kirby RS. Outcomes of robotic assisted radical prostatectomy. Int J Urol. 2009;16(3):244-248.

37. Lim SK, Kim KH, Shin TY, Rha KH. Current status of robot-assisted laparoscopic radical prostatectomy: how does it compare with other surgical approaches? Int J Urol. 2013;20(3):271-284.

38. Mottrie A, Gallina A, De Wil P, Thüer D, Novara G, Ficarra V. Balancing continence function and oncological outcomes during robot-assisted radical prostatectomy (RARP). BJU Int. 2011; 108(6 Pt 2):999-1006.

39. Porpiglia F, Morra I, Lucci Chiarissi M, et al. Randomised controlled trial comparing laparoscopic and robot-assisted radical prostatectomy. Eur Urol. 2013;63(4):606-614.

40. Ramsay C, Pickard R, Robertson C, et al. Systematic review and economic modelling of the relative clinical benefit and cost-effectiveness of laparoscopic surgery and robotic surgery for removal of the prostate in men with localised prostate cancer. Health Technol Assess. 2012;16(41): 1-313.

41. Bolenz C, Freedland SJ, Hollenbeck BK, et al. Costs of radical prostatectomy for prostate cancer: a systematic review. Eur Urol. 2014;65(2):316-324.

42. Rebuck DA, Zhao LC, Helfand BT, et al. Simple modifications in operating room processes to reduce the times and costs associated with robot-assisted laparoscopic radical prostatectomy. J Endourol. 2011;25(6):955-960.

43. Nguyen MM, Das S. The evolution of robotic urologic surgery. Urol Clin North Am. 2004;31(4):653-658, vii.

44. Satava RM, Bowersox JC, Mack M, Krummel TM. Robotic surgery: state of the art and future trends. Contemp Surg. 2001;57(10):489-499.

45. Sahoo SK, Ma W, Labhasetwar V. Efficacy of transferrin-conjugated paclitaxel-loaded nanoparticles in a murine model of prostate cancer. Int J Cancer. 2004;112(2):335-340.

46. Shergill IS, Rao A, Arya M, Patel H, Gill IS. Nanotechnology: potential applications in urology. BJU Int. 2006;97(2):219-220.
Robotic Surgery: Research and Reviews

\section{Publish your work in this journal}

Robotic Surgery: Research and Reviews is an international, peer reviewed, open access, online journal publishing original research, commentaries, reports, and reviews on the theory, use and application of robotics in surgical interventions. Articles on the use of supervisory-controlled robotic systems, telesurgical devices, and shared-control systems are

\section{Dovepress}

invited. The manuscript management system is completely online and includes a very quick and fair peer review system, which is all easy to use. Visit http://www.dovepress.com/testimonials.php to read real quotes from published authors. 